\title{
Féeries
}

Études sur le conte merveilleux, XVII $-\mathrm{XIX}{ }^{\mathrm{e}}$ siècle

\section{Le merveilleux comme expérience initiatique (Godard de Beauchamps, Sainte-Foy d'Arcq et Marmontel)}

The Marvellous as Initiatory Experience (Godard de Beauchamps, Sainte-Foy d'Arcq and Marmontel)

\section{Magali Fourgnaud}

\section{OpenEdition \\ Journals}

Édition électronique

URL : http://journals.openedition.org/feeries/1731

DOI : 10.4000/feeries. 1731

ISSN : 1957-7753

\section{Éditeur}

UGA Éditions/Université Grenoble Alpes

\section{Édition imprimée}

ISBN : 978-2-37747-075-4

ISSN : $1766-2842$

\section{Référence électronique}

Magali Fourgnaud, « Le merveilleux comme expérience initiatique (Godard de Beauchamps, SainteFoy d'Arcq et Marmontel) », Féeries [En ligne], 15 | 2018, mis en ligne le 14 février 2019, consulté le 08 septembre 2020. URL : http://journals.openedition.org/feeries/1731 ; DOI : https://doi.org/10.4000/ feeries. 1731

Ce document a été généré automatiquement le 8 septembre 2020

(c) Féeries 


\section{Le merveilleux comme expérience initiatique (Godard de Beauchamps, Sainte-Foy d'Arcq et Marmontel)}

The Marvellous as Initiatory Experience (Godard de Beauchamps, Sainte-Foy

d'Arcq and Marmontel)

Magali Fourgnaud

Les anthropologues et les folkloristes ${ }^{1}$ ont depuis longtemps établi des liens entre les contes de la tradition et les rituels d'initiation. Comme l'a montré Mircea Eliade, les reprises littéraires de structures et de motifs qui rappellent les épreuves et les cérémonies des religions primitives témoignent d'un besoin profond de l'être humain d'être relié à lui-même, à la société à laquelle il appartient et à l'univers. Pour l'historien des religions, la pérennité et le renouvellement de ces scénarios archaïques suggèrent que «l'initiation [est] un processus indispensable à tout essai de régénération totale, à tout effort de transcender la condition naturelle de l'homme ${ }^{2}$ ». Si dans la plupart des contes les éléments initiatiques sont " camouflés $^{3}$ ", les trois récits que je me propose d'étudier mettent en scène explicitement l'initiation du personnage principal, si l'on entend par initiation une «modification radicale du statut religieux et social du sujet à initier ${ }^{4} »$. En outre, ces contes, écrits entre 1737 et 1765 , relèvent de ce que Jean-Paul Sermain a défini comme des "métafictions ${ }^{5}$ ", dans la mesure où la mise en abyme sur laquelle ils reposent conduit le lecteur à s'interroger sur sa propre réception de la fiction. Les trois personnages principaux ont en commun de vivre une expérience merveilleuse qui a pour effet de dissiper leurs illusions et de les amener à penser par eux-mêmes. Par leur dimension métatextuelle, ces trois contes à visée philosophique ${ }^{6}$ nous permettent d'observer les retentissements physiques et moraux du merveilleux, et de voir comment cette confrontation à ce qui échappe à la rationalité humaine participe, paradoxalement, à l'éclairement des consciences. Dans son ouvrage consacré aux Gestes d'humanités, Yves Citton établit un lien entre expériences esthétiques et mysticisme, compris comme initiation à des mystères : «[...] c'est toute la conception de l'art mise en place au cours des deux derniers siècles qui 
reproduit la structure contradictoire d'un mystère indicible dont l'expérience sensible et extatique nous exalte à un niveau supérieur d'existence ${ }^{7}$. " En quelque sorte les contes de Pierre-François Godard de Beauchamps (Funestine ${ }^{8}$, 1737), de Philippe de Sainte-Foy d'Arcq (Le Palais du silence, conte philosophique ${ }^{9}$, 1754) et de Jean-François Marmontel (Le Mari Sylphe ${ }^{10}$, 1765) illustrent cette fonction initiatique de la fiction.

Ces récits proposent trois modalités différentes du merveilleux : le merveilleux en tant que catégorie esthétique, les merveilles c'est-à-dire les phénomènes féeriques ou surnaturels, et la merveille exprimant le retentissement subjectif que provoque tout événement étonnant. Seul le conte de Beauchamps relève véritablement de la première catégorie car il instaure d'emblée un cadre fictionnel féerique: Funestine, princesse d'Australie, naît sous les auspices néfastes de fées malfaisantes mais trouvera chez le génie Clair-Obscur un nouveau protecteur. Tous les événements rapportés dans le conte relèvent de la logique féerique : apparition-disparition instantanée de monstres, déplacements immédiats des fées et du génie, vertus magiques des éléments (l'eau du fleuve par exemple a le pouvoir de dissiper les faiblesses humaines et de transformer en héros ceux qui s'y baignent). Paradoxalement, la métamorphose des deux personnages principaux (Funestine et Formose, le fils de Clair-Obscur qui lui est destiné) ne relève pas du merveilleux. Née laide, méchante et tyrannique, Funestine se transforme au terme du conte en une belle et lumineuse princesse. Mais cette modification n'est pas le fruit d'une intervention féerique. Certes deux fées-allégories, Vertu et Imagination, aidées par une troisième, la fée Docilité, aident la princesse Funestine à se défaire de ses préjugés et à s'engager dans un travail sur elle-même, mais c'est surtout l'interprétation d'un songe allégorique qui lui permet de se libérer des obstacles qui l'empêchent d'être en harmonie avec elle-même et avec les autres : le déchiffrement de l'histoire, suivi d'un retour sur sa propre situation, concourt à l'acquisition d'une meilleure connaissance de soi. À la fin de cet apprentissage, Funestine devient belle, mais sa beauté est avant tout morale : elle a su déchiffrer son cœur, comme un livre, afin de pouvoir s'ouvrir aux autres.

2 Dans le conte de Sainte-Foy D'Arq, Le Palais du silence, conte philosophique, la démarche herméneutique conduit également le personnage principal à davantage de lucidité. Le cadre fictionnel est ici historique, comme le signale la «Dissertation historique et critique » qui fait office de préface : « On ne doit pas regarder le Palais du Silence tout-àfait comme une fiction, puisqu'on y retrouve des traces de l'Histoire ${ }^{11}$.» Le récit, attribué à Cadmus de Millet, rend compte des tensions politiques et des séditions qui ont caractérisé l'établissement des colonies grecques en Asie mineure. Le jeune prince, Iphis, fils de Clidème, historien et gouverneur de Cnide, raconte comment il a été manipulé par Protas et par Mezronime, la prêtresse de Vénus, et s'est retrouvé prêt à organiser une faction pour renverser son propre père. C'est là qu'intervient l'événement merveilleux : à la suite d'un tremblement de terre, Iphis se retrouve dans une grotte, puis dans le palais du silence dédié à Harpocrate. Dès qu'il pense à un membre de son entourage, l'image de ce dernier est projetée sur les parois du sanctuaire. Iphis assiste ainsi aux conjurations menées par Protas contre lui et découvre l'hypocrisie de Mezronime, mais aussi la fidélité de Théone et de son ami Ménocles qui deviendra son "mentor » une fois qu'il aura recouvré le pouvoir. Si les projections de ces images constituent bien un phénomène merveilleux ${ }^{12}$, l'effet qu'elles produisent est l'éclairement de la conscience du personnage: «[...] profitons du moment où je puis me précautionner contre les fausses apparences de la cour [dit 
Iphis]. Sans le miracle qui s'opère comment n'y serais-je pas trompé ${ }^{13}$ ? " La grotte et le palais du silence s'apparentent ainsi à une camera obscura, c'est-à-dire à une "machine anamorphique ${ }^{14}$ ", une boîte secrète qui, par un « petit trou », montre l'autre aspect du monde, sa face cachée. La palingénésie du personnage repose sur les connaissances qu'il a acquises dans ces lieux (il observe également une querelle entre Pythagore et Thalès) et sur le développement de ses capacités d'examen ${ }^{15}$.

3 Enfin, on pourrait dire concernant le conte de Marmontel, Le Mari Sylphe, qu'il s'agit cette fois d'une merveille sans merveilleux. En effet, ici aucune fée, aucun élément magique, aucun sylphe contrairement à ce qu'annonce le titre. En revanche, le stratagème mis en place par Volange, qui se fait passer pour un être merveilleux auprès de son épouse Élise, a pour effet de la désillusionner. Élevée dans la croyance que hommes et femmes ne sont que des ennemis, Élise est persuadée qu'elle ne s'est mariée que par complaisance et refuse d'éprouver quelque sentiment pour son époux. Friande de fictions, elle se passionne pour les fables des sylphes si bien qu'elle finit par en rêver et voir à l'opéra l'incarnation de ses fantasmes. Volange, son mari, mis dans la confidence par la servante Justine, décide alors de mettre en place un stratagème pour dissiper les illusions de sa femme : il se fera passer pour un sylphe au moment de son coucher. Touché par la fidélité de son épouse qui résiste aux avances du « roi des airs ", Volange lui révèle le tour. Paradoxalement, cette démystification est vécue comme un " enchantement ${ }^{16}$ ". La mise en scène s'apparente donc à une merveille, à une " chose qui étonne », conformément à l'étymologie.

4 Ces trois contes, dont les dispositifs narratifs reprennent des éléments des rites initiatiques religieux, montrent que le détour par la représentation symbolique et par l'éveil des sens conduit à un éclairement de la conscience. La vérité qu'il s'agit d'atteindre n'est plus ici d'origine divine, elle est intérieure à l'être humain. Ces fictions attestent de la manière dont les conteurs des Lumières se sont approprié les rites et les mythes religieux, contribuant ainsi à leur laïcisation.

Ce qui frappe dans ces trois contes, c'est l'importance accordée au lexique religieux, mêlant ainsi dans un élan syncrétique les traditions et les rites. Le conte de Sainte-Foy d'Arcq fait ainsi clairement allusion aux rituels antiques : Iphis cherche à convaincre son père de faire appel aux oracles pour choisir sa future épouse; des sacrifices et des offrandes sont offerts à Vénus dont le temple est gardé par la prêtresse Mezronime. Les hommes se considèrent comme les objets des dieux, leur sort dépendant des humeurs et des attentions divines à leur égard. Le père d'Iphis met en garde son fils contre les fausses apparences de l'Amour : « [...] gardez-vous de vous laisser initier à ses mystères. [...] attendez que ce Dieu, de qui dépend le vrai bonheur du monde soit apaisé pour nous, et revienne prendre soin, lui-même, de votre destinée ${ }^{17}$.» Le cheminement d'Iphis se fait ensuite sous les auspices du dieu Harpocrate, dont le temple et la statue sont représentés sur le frontispice du conte. Le parcours est véritablement initiatique, comme l'illustre son accès au palais, par un " petit escalier de marbre »:

L'obscurité totale où je me trouvai longtemps, me faisait chanceler à chaque degré que je heurtais : enfin j'arrivai sans accident, jusqu'au faîte de ce pavillon. Une porte basse s'ouvrit d'elle-même, et me rendit la lumière. Je ne sais encore ce qui la produisit. J'entrai dans un cabinet de figure ovale, entièrement revêtu d'acier parfaitement poli, au travers duquel il était impossible qu'aucun astre portât ses rayons. Je me reposai sur une estrade, placée au milieu de ce cabinet ${ }^{18}$.

6 L'obscurité des lieux et le mutisme auquel le personnage est réduit ${ }^{19}$ créent l'effet d'une mort symbolique, comme le formule le personnage lui-même: «[...] ce fut alors, que 
j'éprouvai tout ce qu'une mort lente, obscure et certaine, peut inspirer d'horreur ${ }^{20}$. » L'intensité des affects ressentis dans le temple le laissent "dans un état inexprimable » : «Un voile épais venait de se déchirer devant mes yeux : je croyais pour ainsi dire penser et sentir pour la première fois ${ }^{21}$. " La concomitance des deux procès, "penser" et "sentir», est particulièrement significative : l'éveil de la conscience d'Iphis - comme celle de Funestine et d'élise - nécessite une initiation par les sens. Cette renaissance symbolique a des effets sur le plan individuel et collectif dans la mesure où la dissipation des illusions du personnage renforce le lien social de la communauté rassemblée autour de lui. De retour chez lui, Iphis, par exemple, a changé complètement de comportement envers ses semblables : «Je marquai de la bonté à tous les courtisans, de la bienveillance à quelques-uns et de l'empressement à quelques autres, selon les lumières que j'avais reçues des murs d'acier ${ }^{22}$.» La configuration, qui s'apparente ici à un "espace initiatique ${ }^{23}$ ", symbolise donc la palingénésie du personnage : l'épreuve, au cours de laquelle Iphis est centré sur lui-même, lui permet paradoxalement de s'ouvrir aux autres ${ }^{24}$. Dans le conte de Godard de Beauchamps, Funestine connaît une évolution similaire: odieuse à l'égard de ses servantes, colérique, considérée comme un "monstre ${ }^{25}$ " par son propre père, la princesse apprend, grâce au dispositif imaginé par le Génie et par les fées, à mesurer son comportement à l'égard d'autrui et à faire preuve de davantage de prudence et d'humilité : « [e]n garde contre elle-même, le souvenir du passé la rendait attentive sur le présent, et timide sur l'avenir ${ }^{26}$." Cette métamorphose morale est le fruit d'un parcours difficile. Comme Iphis, Funestine est isolée du milieu familial, d'abord enfermée dans un palais magnifique et immense, protégé des maladies et des intempéries, puis, sur les conseils de la fée Vertu, dans un lieu plus modeste, « une petite maison où Funestine serait moins distraite par la diversité des objets, serait plus capable d'attention et de recueillement ${ }^{27}$ ». La jeune princesse prend conscience de ses travers également au sein d'un temple, dédié cette fois à la Vertu, dans lequel elle s'imagine pénétrer au cours d'un songe. Comme dans le conte de Sainte-Foy d'Arcq, l'accès au lieu sacré est périlleux: «[...] ma fermeté prit le dessus, je franchis les obstacles, j'arrivai à l'enceinte du temple, le visage meurtri, les mains et les pieds tout en sang; je souffrais des douleurs inexprimables, je pleurais amèrement, je ne pouvais plus me soutenir ${ }^{28}$.» Puis vient l'épreuve de purification et de mort symbolique : «Une femme éclatante de lumière me prit par la main, [...] on m'ôta mes habits, on me plongea dans une cuve pleine d'une liqueur si forte et si spiritueuse, que je n'en pus soutenir l'effet, un feu dévorant me consumait, je crus qu'une flèche me perçait le cœur, je perdis connaissance ${ }^{29}$. " De manière plus implicite, le conte de Marmontel garde lui aussi des traces des rituels religieux : les «apparitions » du sylphe ont lieu la nuit, car «[1]es tableaux de l'imagination ne sont jamais si vifs que dans l'obscurité profonde $^{30} »$; l'émotion d'Élise à l'écoute de la voix de l'être fantasmé est telle qu'elle perd elle aussi connaissance : "Ah! je me meurs; je viens de l'entendre. Rappelle-moi s'il se peut à la vie [dit-elle à Justine] ${ }^{31}$. » Par conséquent, les trois contes reprennent de manière plus ou moins explicite des éléments des scénarios initiatiques issus aussi bien de la tradition antique que des rituels chrétiens. En effet, la réclusion, le silence, l'éloignement des proches évoquent également les exercices spirituels imposés par les Jésuites aux novices, tels que les rapporte Louis-René De Caradeuc de la Chalotais :

Un jeune homme est renfermé seul dans une chambre, privé de livres et éloigné de tout bruit, afin qu'il n'ait aucune distraction; il doit faire des méditations [...]. Lorsqu'il médite sur l'enfer, il doit voir une plaine enflammée, des âmes brûlées 
dans des corps de feu, entendre des hurlements, des blasphèmes ; s'imaginer qu'il éprouve, par l'odorat et par le goût, les sensations les plus rebutantes. Chaque novice est averti [...] qu'il doit être frappé des objets comme s'il les voyait, qu'il doit voir par les yeux de l'imagination, goûter par le goût de l'imagination, etc. Il y avait même autrefois une chambre de méditations où l'on mettait des tableaux pour que l'imagination fût aidée ${ }^{32}$.

Dans le conte de Sainte-Foy d'Arq, Iphis est placé dans une situation similaire. Le sanctuaire dans lequel il pénètre présente en effet à la vue du jeune prince des peintures "historiques ou morales, chrétiennes", édifiantes et allégoriques ${ }^{33}$ et les images qui sont projetées par magie sur les parois du « cabinet d'acier » sont assimilées à de véritables «tableaux", des "scènes" qui bouleversent profondément le spectateur. Dans le conte de Beauchamps, l'intérieur du temple est également orné d'allégories mystérieuses que Funestine considère avec attention «sans pouvoir en pénétrer le sens ${ }^{34} »$. La reprise de ces motifs initiatiques témoigne du processus de laïcisation des mythes et rites religieux : comme l'a montré Paul Bénichou, «l'ancien système religieux a été concurrencé ou supplanté, du XVIII ${ }^{\mathrm{e}}$ au $\mathrm{XIX}^{\mathrm{e}}$ siècle, par une foi dans laquelle l'homme, et non Dieu, tendait à occuper la première place ${ }^{35}$ ».

En effet, dans les trois contes étudiés, il n'est aucunement question de révélation divine ou de vérité extérieure à l'être humain. Iphis, par exemple, est surpris de la lumière qui surgit de l'intérieur du temple et ne l'attribue à aucune instance supérieure. C'est qu'il s'agit surtout de trouver une beauté morale intérieure : celle-ci, dit la fée Imagination à Funestine, "est au-dedans de vous, travaillez à la développer; elle se laisse trouver quand on la cherche [...]. Je ne puis que vous montrer le chemin qui conduit à son temple, Vertu vous en ouvrira le sanctuaire, si vous suivez ses conseils, attendez tout des Dieux, et de vous-même, je ne vous révèle point l'avenir ${ }^{36}$ ». Dans le conte de SainteFoy d'Arcq, Iphis reconnaît que seul un travail sur lui-même peut lui permettre d'atteindre une vérité morale : «[...] il n'était réservé, qu'à ma propre expérience, de m'instruire et de me ramener à la vertu. Les préceptes les plus sages ne me défendirent point de l'ivresse des passions ${ }^{37}$.» Mettant l'expérience et la réflexivité au premier plan, ces contes témoignent des bouleversements philosophiques de la première moitié du XVIII ${ }^{e}$ siècle : dégagée de tout essentialisme et de toute transcendance, la morale se trouve désormais au cœur de l'expérience humaine, grâce notamment à la sympathie, que Jaucourt définit comme "cette conformité de qualités naturelles, d'idées, d'humeurs et de tempéraments ${ }^{38}$ ». Cette analogie de sentiments et de sensations permet à l'homme de s'imaginer à la place d'autrui, condition nécessaire à l'ouverture du champ de conscience.

9 En effet, les modifications profondes d'ordre moral que connaissent les personnages des trois contes sont les conséquences des retentissements physiques et émotionnels suscités par les images (le terme désignant aussi bien les projections sur les parois du palais du silence, le songe de Funestine que les fantasmes d'Élise). Dans Le Palais $d u$ silence, Iphis, ému par le courage et la dignité de son père, «s'élanc[e] avec transport pour [se] jeter à ses genoux ». Puis à la vue des conjurés, il s'exclame : «L'étonnement et l'horreur dont je fus pénétré, me laissèrent à peine l'usage de mes sens, pour entendre cette funeste conversation. J'oubliai plus d'une fois l'obstacle qui dérobait ces monstres à ma fureur et je me précipitai le poignard à la main pour les immoler ${ }^{39}$. La rupture de ce qu'on nommerait aujourd'hui le "quatrième mur", séparant la représentation du spectateur, met en évidence la puissance de l'émotion provoquée par l'illusion de voir la scène se dérouler sous ses yeux ${ }^{40}$. Suit alors l'énumération des 
sentiments qui agitent le prince, mû tour à tour par la crainte, la honte, le remords, la rage, le regret. Le conte de Beauchamps met également en scène la puissance des affects provoqués par l'imagination, allégorisée à travers la fée du même nom. Celle-ci s'avère la seule capable de rendre la princesse Funestine "attentive, sensible », de la retenir par « un pouvoir invisible» et de provoquer en elle « des mouvements qu'elle n'avait point encore éprouvés ${ }^{41} »$ :

Ce discours [de la fée] fit une si vive impression sur Funestine, qu'elle était comme hors d'elle-même. On ne passe point sans agitation d'un état à un autre; la joie imprévue affecte autant que la douleur, la sienne était remplie de trouble et d'inquiétude, elle regardait Imagination, elle en détournait les yeux; elle soupirait, elle lui baisait les mains avec transport, elle voulait lui marquer sa reconnaissance, elle ne trouvait point de termes pour l'exprimer ; ses pensées se confondaient, elles ressemblaient aux rêves d'un malade. Imagination s'applaudissait des mouvements que sa présence excitait dans l'âme de Funestine, elle voyait avec plaisir toute l'étendue de sa sensibilité ; quand elle eut assez joui, elle la rendit à elle-même ${ }^{42}$.

10 L'isotopie du mouvement souligne les effets physiques et la force motrice de l'imagination. Cette scène théâtralise ainsi l'impact de l'esprit sur le corps, la fée explicitant d'ailleurs le lien qui s'établit entre l'imagination, l'âme et « l'étendue de [1]a sensibilité », comprise à la fois dans son sens corporel (faculté de sentir) et moral (disposition à être ému, touché).

11 Ainsi, la scénographie spéculaire dans laquelle les personnages principaux des trois contes sont placés fait naître en eux un sujet moral, un «spectateur impartial », tel que l'a conceptualisé Adam Smith dans sa Théorie des sentiments moraux (1759). Selon le philosophe écossais, le sentiment moral nécessite, pour se développer, le recours à l'imagination et à une mise en scène rassemblant trois personnages: celui qui fait l'action, celui qui la subit ou en bénéficie et un témoin désintéressé, cherchant à mesurer la justesse de l'action. Or nous ne pouvons juger des sentiments et des sensations d'autrui qu'en fonction des nôtres. Partant, seule l'imagination nous permet de conjecturer sur ce que nous ressentirions si nous étions à la place des deux acteurs observés. Cette analogie de sentiments, que Smith désigne par le terme de «sympathie» (fellow-feeling), nous conduit à modifier nos comportements en fonction des retentissements que nous imaginons chez autrui, selon nos propres perceptions. Le sujet moral est celui qui saura mettre en adéquation ses actions et ses sentiments avec un certain «point de convenance » qui nous conduit à ressentir naturellement ce qui est juste :

L'horreur que l'atrocité d'une telle conduite [à savoir l'injustice d'un tyran] nous inspire, le plaisir que nous prenons en apprenant qu'elle a été châtiée comme il convenait, l'indignation que nous ressentons si elle échappe à ces légitimes représailles, en un mot la conscience que nous avons de son démérite, de la convenance qui se trouve à faire du mal à la personne qui en est coupable, et à la faire souffrir à son tour, naît de l'indignation sympathique qui bouillonne naturellement dans le sein du spectateur, toutes les fois qu'il rapporte pleinement à lui-même la situation de celui qui souffre ${ }^{43}$.

12 Le conte de Sainte-Foy d'Arcq met en scène cette contagion des affects capable de déclencher un comportement moral. Lorsqu'Iphis voit apparaître la fidèle Théone, en proie à un douloureux dilemme au moment où elle s'apprête à dénoncer les membres de sa famille pour sauver le roi, le jeune prince s'exclame :

Le désordre de sa parure, la pâleur de son visage baigné de pleurs, ses mains et ses yeux qu'à chaque instant elle élevait au ciel, sa démarche incertaine et précipitée caractérisaient le trouble de son âme. La mienne en fut émue : je ne savais à quoi 
attribuer l'extrême douleur dont elle donnait des marques si vraies et si touchantes, je craignais de m'en éclaircir ; quelle peine encore pour moi, si la cause en eut été funeste ou criminelle! Il me devenait presqu'également douloureux, que Théone pût être infortunée ou coupable ${ }^{44}$.

Il est intéressant de voir comment le jeune prince lit et ressent simultanément la douleur de la jeune femme uniquement par ses gestes. L'émergence d'un sujet moral, autonome, capable de penser par lui-même semble nécessiter le recours à une scénographie théâtrale, ce que montre également le conte de Marmontel, Le Mari Sylphe. Le lexique de la comédie émaille en effet l'ensemble du texte et la chambre d'élise, où apparaît le sylphe (nommé Valoé), est décrite comme une scène de théâtre, sur laquelle la servante Justine et Volange (le mari) jouent une farce à Élise pour la désenchanter de ses illusions. La théâtralité du conte est renforcée par le fait que Justine feint de ne pas entendre les apartés de sa maîtresse et de ne pas voir la substitution du tableau (offert soi-disant par le sylphe) au miroir ; le jardinier nie être à l'origine de la réalisation du magnifique bosquet, renforçant ainsi l'illusion d'un phénomène merveilleux. La mise en scène semble donc mystifier le personnage pour mieux le ré-enchanter: "C'est à présent, dit Élise en se jetant dans les bras de son époux, c'est à présent que je suis enchantée, et j'espère que la mort seule détruira cet enchantement ${ }^{45}$. " Comme Iphis, Élise renaît à elle-même, la dissipation des illusions engendrant un mouvement d'amour sincère et de joie. Mais on peut également lire dans le conte de Marmontel l'évolution morale du mari. En effet, le dispositif inventé lui permet de devenir le confident de sa femme. Cette dernière lui révèle ainsi les contraintes de la condition féminine : «[...] je vis avec lui comme avec un homme : dans la défiance et la crainte que nous inspire naturellement un sexe né l'ennemi du nôtre. On m'a donnée à lui sans me consulter, j'ai suivi mon devoir et non pas mon penchant ${ }^{46}$. » De telles révélations, que les relations maritales codifiées par la société ne permettaient certainement pas de faire aussi librement, déclenchent chez VolangeValoé un mouvement de sympathie, au sens où la définit Smith :

Dès que votre mari vous aura déplu, vous m'en instruirez, et dans l'instant, le regret, le reproche, se saisiront de son âme, et il n'aura de paix ni avec moi, ni avec lui-même, qu'il n'ait expié à vos genoux le déplaisir qu'il vous aura causé. Je ferai plus, je lui inspirerai tout ce que vous m'inspirez à moi-même. Ainsi l'esprit de votre Sylphe animera votre mari, et vous sera présent sans cesse. - Voilà, dit Élise enchantée, le seul moyen de me le faire aimer ${ }^{47}$.

Le passage suggère la lucidité d'Élise, qui tout en feignant d'être leurrée par son entourage, participe à l'éclairement de la conscience de son mari. Comme pour Iphis, l'émergence d'un sentiment moral passe par l'imagination et par un dispositif théâtral : pour comprendre ce que ressent son épouse, Volange doit jouer le rôle de confident et s'imaginer à la place de l'être rêvé. C'est cette reconnaissance mutuelle qui fait naître un sentiment d'amour chez Élise, ce que Smith formule ainsi :

Qu'il semble aimable, celui dont le cœur plein de sympathie paraît faire écho à tous les sentiments des personnes avec lesquelles il converse, qui s'afflige de leurs peines, qui ressent leurs injures, qui se réjouit de leur prospérité ! Lorsque nous nous mettons à la place de ses compagnons, nous partageons leur reconnaissance, et nous sentons tout le réconfort que leur doit procurer la tendre sympathie d'un ami si affectionné ${ }^{48}$.

15 Si l'élargissement de la conscience des trois personnages évoqués est la conséquence de l'exacerbation de leurs sens et de l'extension de leur sensibilité, il passe également par une démarche herméneutique et analogique. Une fois passé le moment de stupeur, les 
personnages s'engagent en effet dans une véritable enquête : Iphis souhaite « démêler la conduite de ceux auxquels [il était] attaché ${ }^{49} "$, les images qui apparaissent au fil de ses pensées sont l'objet d'un « examen » qui lui permet de s' « instruire ${ }^{50}$ » du cœur des hommes et ainsi de le ramener «dans le chemin de la sagesse, de la gloire et du bonheur $^{51}$ ». Cette démarche critique dans laquelle s'impliquent les trois personnages vise à dissiper leurs propres illusions. En effet, si ces trois personnages sont "monstrueux", c'est qu'ils sont tous les trois bernés par les fausses apparences, enfermés dans leurs représentations: à Funestine qui, contente d'elle-même, fait régner la terreur dans son palais, le conteur annonce que «[1]e temps approchait où son illusion devait se dissiper ${ }^{52}$ »; Élise s'est laissée enfermer dans les romans et les contes merveilleux car « ces brillantes chimères avaient tout le charme de la vérité ${ }^{53}$ "; Iphis reconnaît également que « le goût du plaisir et les prestiges de l'orgueil portèrent dans [s] on âme les plus séduisantes illusions ${ }^{54}{ }$; les images ont provoqué ainsi chez lui "l'humiliante découverte de la source de [s]es égarements et de [s]es malheurs dans [s]a folle vanitéf5 $»$. Dès lors, ces trois personnages cherchent à s'émanciper des passions aveuglantes et ce processus nécessite le recours à une lecture analogique. La première fois que Funestine découvre sa laideur, c'est à l'écoute du conte que lui fait le conteur Quart-d'-heure : il peint la belle princesse de l'histoire sous les traits d'Imae dont il est amoureux, déclenchant ainsi la jalousie furieuse de Funestine. C'est que le cheminement qui conduit à la sagesse est long et douloureux, comme le symbolisent les épreuves physiques auxquelles la princesse est confrontée : "Qu'il en coûte pour détruire les premières impressions de l'habitude ! [s'écrie le narrateur de Funestine] Le combat fut pénible, la victoire douteuse, enfin la raison prit le dessus ${ }^{56}$.» Observer et accepter les contradictions humaines, remettre en question ses catégories de pensées, en somme, philosopher nécessitent un changement radical de perspective, que symbolise le passage par un lieu silencieux, souterrain et obscur. Telle est d'ailleurs la lecture allégorique que propose Funestine de son propre rêve :

[C]et homme couché par terre est le Découragement; ces spectres sont les Préjugés; ces degrés sont les épreuves; cette porte du temple fermée, qui ne s'ouvre qu'après une longue attente montre qu'on n'y peut entrer que par la persévérance : ces emblèmes sont les attributs, et les mystères de la Vertu, que le Temps, figuré par ce vieillard, peut seul faire comprendre; [...] cette femme éclatante de lumière est la Raison ${ }^{57}$.

16 In fine, le déchiffrement auquel procède Funestine, et qui s'exprime ici par le parallélisme des constructions attributives, la libère de ses préjugés et de ses monstres intérieurs. Plus précisément, la fée Imagination l'invite à se « reconnaître » dans cette fiction nocturne, à établir des " rapports avec la situation où [elle est] ${ }^{58}$ ». Aucun sens extérieur à trouver ici sous le voile de l'histoire : la signification n'émerge que dans le processus même de la lecture, qui consiste dès lors à établir des rapports d'analogie entre le rêve et la situation vécue : «Ah! s'écria-t-elle, mon rêve s'accomplit, et je n'ai plus besoin qu'on me l'explique ${ }^{59}$." Le conte de Beauchamps témoigne ainsi du renouvellement, dans la première moitié $d u$ xvIII ${ }^{e}$ siècle, du pacte de lecture qui d'allégorique devient analogique ${ }^{60}$.

17 En mettant en scène l'évolution morale du personnage, rendue possible à la fois par l'intensité des affects provoqués par le phénomène merveilleux et par son déchiffrement, les contes ici étudiés mettent en place une analogie : à l'instar de l'initiation des personnages, l'effet visé de leur lecture est bien l'élargissement de la conscience morale du lecteur, favorisé par l'éveil des sens et par la démarche 
herméneutique. En effet, ces trois contes placent le lecteur dans la situation d'un "spectateur impartial », témoin de l'émergence d'un sujet moral. Cette mise en abyme confère aux contes une portée morale car elle favorise une sorte de communion entre l'œuvre et le lecteur :

C'est que, durant l'illusion votre âme et la leur [celle des auteurs] n'en font qu'une : ce sont comme deux instruments organisés de même, et accordés à l'unisson. Mais si l'âme du Poète ne s'est pas montée au ton de la Nature, le personnage auquel il a communiqué ses sentiments et son langage, n'est plus dans la vérité de la situation et de son caractère; et vous, qui vous mettez à sa place mieux que n'a fait le Poète, vous n'êtes plus d'accord avec lui ${ }^{61}$.

18 L'expérience esthétique se rapproche ici des expériences morales décrites par Smith, la "Nature» servant de "point de convenance " à partir duquel nous jugeons de la vraisemblance et de la vérité morale de l'œuvre. Pour autant, un écart est sans cesse maintenu entre la chose évoquée et son image :

Chacun de nous a, comme le Poète, la faculté de se mettre à la place de son semblable, et l'on s'y met réellement tant que dure l'illusion. On pense, on agit, on s'exprime avec lui, comme si on était lui-même; et, selon qu'il suit nos pressentiments ou qu'il s'en écarte, la fiction qui nous le présente est plus ou moins vraisemblable pour nous ${ }^{62}$.

19 Marmontel insiste ici sur le " comme si ». L'illusion déclenche la sympathie du lecteur, au sens que lui donne Smith, mais il reste toujours conscient qu'il s'agit d'une illusion ${ }^{63}$. Le Mari Sylphe met d'ailleurs en scène cette " feintise partagée ${ }^{64}$ » qu'instaure la fiction littéraire. Dès le début, Élise est consciente que le sylphe qui lui apparaît n'existe pas. À Justine, sa servante, qui lui oppose un discours rationalisant, elle s'écrie :

Tu me désespères: est-ce là m'aimer que de m'envier, que de vouloir détruire la plus flatteuse illusion ! car c'en est une, je dois le croire, et je ne suis pas un enfant... Cependant l'odeur des roses ! ... oui, je la sens, rien n'est plus réel, et ce n'est pas la saison des fleurs. - Que voulez-vous que je vous dise, madame ? Tout le désir que j'ai de vous plaire ne peut me faire croire qu'un songe soit une vérité. - Hé bien, mademoiselle, ne le croyez pas. Préparez ma toilette et que je m'habille. Je suis dans un trouble, dans une émotion dont je rougis, et que je ne saurais calmer ${ }^{65}$.

Élise formule ici tout le paradoxe de la fiction littéraire ${ }^{66}:$ alors que le lecteur sait que l'histoire qu'il lit est une fiction, ses sens restent en éveil et ses émotions sont bien réelles $^{67}$. Le conte reproduit ainsi son propre mode de fonctionnement. Il en va de même du récit de Beauchamps dans lequel le parcours moral de Funestine se fait au cœur des contes. Le palais des Événements que lui construit le génie Clair-Obscur possède une bibliothèque où «se trouvaient les originaux des ouvrages que devaient faire les D’Aulnoy, les Murat, les Durand [...] [dont les portraits étaient] couronnés de rayons de lumière ${ }^{68}$ ». Cette énumération, qui relève de "l'effet anthologique ${ }^{69}$ »du conte merveilleux, souligne la réflexivité du conte, qui devient dès lors un lieu de réflexion et d'expérimentation de la fiction.

21 Ainsi, à la différence des exercices spirituels des Jésuites, qui cherchent à provoquer chez les novices terreur et soumission à l'ordre, les contes de Beauchamps, Sainte-Foy d'Arcq et Marmontel visent au contraire à éveiller leurs lecteurs et à aiguiser leur perspicacité. Les conteurs des Lumières usent ainsi des techniques de leurs ennemis (recours aux images et aux affects pour déclencher une transformation morale), mais contestent toute clôture dogmatique du sens. Beauchamps s'amuse ainsi à souligner l'incomplétude de son récit, à l'instar du miroir «imparfait ${ }^{70}$ » du génie Clair-Obscur, miroir que l'ouvrier, foudroyé par la mort, n'a pas eu le temps de finir. Il en est 
d'ailleurs de même du récit lui-même : «[...] je suis obligé de rompre l'histoire, parce que le reste du manuscrit est d'une autre main, et que les caractères m'en sont inconnus. Je présume que l'Auteur, prévenu par la mort, n'a pu l'achever ${ }^{71}$. " La pluralité des auteurs (l'histoire est attribuée tantôt à un gymnosophiste indien, tantôt à l'historiographe Albupipargamos) est l'expression de l'humilité auctoriale et d'un refus d'enfermer l'histoire dans une signification univoque : «[...] rien de suspect dans mes allégories [précise d'ailleurs le narrateur], rien qui soit susceptible de sinistres applications, rien dans mes portraits qui puisse blesser la religion, ou la délicatesse des grands que je respecte ${ }^{72}$.» La complexité formelle du récit, qui se rapproche de l'esthétique rococo, contribue également à diffracter le sens, à l'image de la figure singulière qui trône au cœur du Palais des Événements : «[...] regardée d'un certain sens, elle représentait un bouc avec des pieds de mulet, la tête d'un chat-huant, et la gueule d'un dogue affamé qui mordait ceux mêmes qui lui donnaient à manger. Regardée sous un autre point de vue, c'était un Bonze japonais, tenant d'une main, un coq et de l'autre, un brasier d'où sortait une épaisse fumée ${ }^{73}$.» Ici s'exprime la jubilation $d u$ conteur à créer des chimères et à emporter le lecteur dans un kaléidoscope de symboles contradictoires, assurément bien éloigné des allégories chrétiennes.

L'étude comparée des contes de Beauchamps, de Sainte-Foy d'Arcq et de Marmontel nous a permis d'observer les retentissements physiques et moraux de l'expérience merveilleuse qui s'apparente ici à une initiation. La renaissance symbolique des trois personnages est le produit à la fois de l'intensité des affects provoqués par le phénomène merveilleux et de la démarche herméneutique à laquelle les personnages procèdent pour déchiffrer leur intériorité et le monde qui les entoure. La métatextualité de ces trois contes met en évidence l'effet de mise en abyme: l'expérience que vivent les personnages se rapproche, par analogie, de celle du lecteur, qui lui aussi vit, à travers la lecture de ces contes, une sorte d'expérience initiatique. L'isolement silencieux de la lecture conduit à un recentrement sur soi-même propice à une ouverture à l'autre. En outre, la complexité des formes de ces contes, en particulier celui de Beauchamps, en rend la perception plus ardue et crée ainsi un effet de dépaysement à la fois sensible et conceptuel, nécessaire à la remise en question des préjugés. Enfin, le dispositif narratif de ces contes demande un déchiffrement singulier : il repose sur un pacte de lecture analogique, invitant le lecteur à tisser des réseaux de correspondances et à opérer un retour réflexif sur sa propre situation. In fine, ces contes visent ainsi à construire un lecteur philosophe, capable de penser par lui-même et de s'émanciper des allégories chrétiennes et moralisatrices.

\section{NOTES}

1. P. Saintyves, Les Contes de Perrault et les récits parallèles, Paris, Robert Laffont, 1987 ; «Initiatiques, ils [les contes merveilleux] montrent la voie, ils disent ce qui doit être, sous forme d'image, sans être didactiques ou moraux: il importe d'abord de faire sa vie hors du foyer parental, peu importe que l'on devienne honnête soldat, habile médecin ou rusé voleur (T.653) », 
J. Bru, «Le repérage et la typologie des contes populaires. Pourquoi ? Comment?», Bulletin de liaison des adhérents de l'AFAS, $\mathrm{n}^{\circ}$ 14, automne 1999.

2. M. Eliade, Initiation, rites, sociétés secrètes, Paris, Folio Essais, 1959, p. 246.

3. Ibid., p. 267.

4. Ibid., p. 12.

5. J.-P. Sermain, Métafictions (1670-1730). La réflexivité dans la littérature d'imagination, Paris, H. Champion, coll. « Les Dix-huitièmes siècles ", 2002.

6. M. Fourgnaud, Le Conte à visée morale et philosophique, de Fénelon à Voltaire, Paris, Classiques Garnier, 2016.

7. Y. Citton, Gestes d'humanités, Paris, Armand Colin, 2012, p. 199.

8. P.-Fr. Godard de Beauchamps, Funestine [1737], dans Le Cabinet des fées, ou Collection choisie des contes des fées et autres contes merveilleux, éd. Ch.-J. Mayer, Amsterdam-Paris, 1786, t. 31, p. 5-102. Je remercie Aurélia Gaillard de m'avoir fait découvrir ce conte.

9. P.-A. de Sainte-Foy d'Arq, Le Palais du silence, conte philosophique, Amsterdam, 1754.

10. J.-Fr. Marmontel, Le Mari Sylphe, dans Sylphes et sylphides, éd. M. Delon, Paris, Desjonquères, 1999 (toutes les références renvoient à cette édition).

11. P.-A. de Sainte-Foy d'Arq, ouvr. cité, p. 11.

12. "Je détournai mes regards du merveilleux mur d'acier, pour être plus à moi-même. » Ibid., p. 94.

13. Ibid., p. 112.

14. A. Gaillard, « Anamorphoses ou les lieux d'illusion picturale dans la fiction (contes et romans) au XVIII ${ }^{\mathrm{e}}$ siècle ", dans Locus in fabula : la topique de l'espace dans les fictions françaises d'Ancien Régime, éd. N. Ferrand, Éditions Peeters, Louvain-Paris-Dudley (Ma.), 2004, p. 547.

15. «[...] les savants et les philosophes de profession ne m'en imposaient plus assez depuis la querelle de Pythagore, pour soumettre mon âme sans examen à leur morale de spéculation plutôt que pratique. » P.-A. de Sainte-Foy d'Arq, ouvr. cité, p. 175.

16. J.-Fr. Marmontel, Le Mari Sylphe, ouvr. cité, p. 122.

17. P.-A. de Sainte-Foy d'Arq, ouvr. cité, Première partie, p. 62.

18. Ibid., p. 30.

19. " Je sentis que je ne pouvais former aucun son. » Ibid., p. 11.

20. Ibid., p. 4.

21. Ibid., p. 94.

22. Ibid., p. 246.

23. «Dans cette dernière famille de configurations, l'espace sert à montrer que la vérité n'est pas facile, qu'elle n'est pas dans ce qui est donné d'emblée. Elle est ce qui est lointain, étranger, qu'il faut rapporter, expliquer [...] dans ce qui est caché, profond, qu'il faut atteindre douloureusement parfois. L'espace aide ici le roman à figurer un savoir auquel on n'a accès que dans un processus, par une activité, un faire. " H. Lafon, Espaces romanesques du XVIII siècle, 1670-1820, de Madame de Villedieu à Nodier, Paris, PUF, 1997, p. 143.

24. Après l'ensemble des épreuves et de retour chez lui, il affirme : « [...] je réunis dans mon cœur le repos des mœurs douces, les transports du véritable amour, le goût des plaisirs purs, les délices de l'amitié, et le désir de mériter l'estime de mes concitoyens et de la postérité. » P.-A. de SainteFoy d'Arq, ouvr. cité, p. 270.

25. P.-Fr. Godard de Beauchamps, ouvr. cité, p. 18.

26. Ibid., p. 126.

27. Ibid., p. 67.

28. Ibid., p. 97.

29. Ibid.

30. J.-Fr. Marmontel, Le Mari Sylphe, ouvr. cité, p. 104.

31. Ibid., p. 105. 
32. L.-R. De Caradeuc de la Chalotais, Compte-rendu des constitutions des Jésuites, [Conclusions devant le Parlement de Rennes, 7 décembre 1761], 1762, p. 173-175. (Je remercie Florence Boulerie de m'avoir fait découvrir ce texte.)

33. L'une représente les sœurs Hersé et Agraulos, punies pour avoir succombé à leur curiosité d'ouvrir la corbeille où se situait le fils d'Athéna et d'Héphaïstos ; l'autre Ascalaphe, enfermé aux Enfers sous l'apparence d'une chouette pour avoir dénoncé Perséphone d'avoir rompu le jeûne et Cyané, la nymphe qui s'opposa à l'enlèvement de Perséphone par Hadès et fut transformée en un océan profond: "Enfin on y observait partout le danger ne pas savoir se taire et Harpocrate semblait avoir affecté de montrer, comment il réprime ceux qui l'outragent. » P.-A. de Sainte-Foy d'Arq, ouvr. cité, p. 16.

34. P.-Fr. Godard de Beauchamps, ouvr. cité, p. 101.

35. P. Bénichou, Le Sacre de l'écrivain (1750-1830). Essai sur l'avènement d'un pouvoir spirituel laïque dans la France moderne, Paris, José Corti, 1985, p. 467.

36. P.-Fr. Godard de Beauchamps, ouvr. cité, p. 92-93.

37. P.-A. de Sainte-Foy d'Arq, ouvr. cité, p. 47.

38. Jaucourt, article "Sympathie ", Encyclopédie ou dictionnaire raisonné des sciences, des arts et des lettres, vol. III, t. XV, Readex Microprint/Pergamon Pres, 1969 [Paris, Briasson, 1751-1772], p. 736.

39. P.-A. de Sainte-Foy d'Arq, ouvr. cité, Deuxième partie, p. 65.

40. Dumarsais définit ainsi l'hypotypose : « [...] c'est ce qui fait l'hypotypose, l'image, la peinture ; il semble que l'action se passe sous nos yeux. » Des Tropes ou des différents sens dans lesquels on peut prendre un même mot dans une même langue, Paris, Manucius, 2011, p. 83-84.

41. P.-Fr. Godard de Beauchamps, ouvr. cité, p. 89.

42. Ibid., p. 93-94.

43. A. Smith, Théorie des sentiments moraux, trad. S. Grouchy, marquise de Condorcet, éd. L. Folliot, Payot \& Rivages, Paris, 2016, p. 192-194.

44. P.-A. de Sainte-Foy d'Arq, ouvr. cité, p. 71.

45. J.-Fr. Marmontel, Le Mari Sylphe, ouvr. cité, p. 122.

46. Ibid., p. 109.

47. Ibid., p. 109-110.

48. A. Smith, ouvr. cité, Première partie, Section première, chap. V, p. 75.

49. P.-A. de Sainte-Foy d'Arq, ouvr. cité, p. 69.

50. Ibid., p. 72.

51. Ibid., p. 97.

52. P.-Fr. Godard de Beauchamps, ouvr. cité, p. 35.

53. J.-Fr. Marmontel, Le Mari Sylphe, ouvr. cité, p. 100.

54. P.-A. de Sainte-Foy d'Arq, ouvr. cité, p. 180.

55. Ibid., p. 67.

56. P.-Fr. Godard de Beauchamps, ouvr. cité, p. 113.

57. Ibid., p. 124-125.

58. Ibid., p. 41.

59. Ibid., p. 113.

60. A. Gaillard, «La fable (ou fiction fabuleuse) aux $\mathrm{XVII}^{\mathrm{e}}$ et $\mathrm{XVIII}^{\mathrm{e}}$ siècles entre allégorie et analogie : une écriture du détour ou de la conciliation?", dans S. Cornic et P. Servet (éds), L'Art de la conciliation, Cahiers du GADGES, $\mathrm{n}^{\circ}$ 11, Lyon, 2014, p. 155-172.

61. J.-Fr. Marmontel, Article "Vraisemblance », dans Éléments de littérature, éd. S. Le Ménahèze, Paris, Desjonquères, 2005, p. 1165.

62. Ibid.

63. «[...] celle-ci, comme l'autre, est quelque fois un art trompeur et mensonger; mais en se livrant aux prestiges de la poésie, on sait qu'on est trompé, et on consent à l'être. » Article « Éloquence », dans Éléments de littérature, ouvr. cité, p. 459. 
64. J.-M. Schaeffer, Pourquoi la fiction?, Paris, Seuil, 1999, p. 156.

65. J.-Fr. Marmontel, Le Mari Sylphe, ouvr. cité, p. 104.

66. Pour Nicolas Veysman, le conte de Marmontel représente ainsi «la mise en fiction de l'échange littéraire entre un auteur illusionniste et un lecteur crédule qui, comme Élise, croit à l'existence d'une voix sans corps - le narrateur - prenant ici les traits de Valoé ». N. Veysman, «Le féerique moral dans les contes moraux de Marmontel », Féeries, n 4, 2007, p. 231.

67. Élise vit en ce sens une expérience similaire à celle du spectateur de théâtre, « demi-illusion » que Marmontel définit comme « cette erreur continue et sans cesse mêlée d'une réflexion qui la dément, cette façon d'être trompé et de ne pas l'être ». Il prend alors l'exemple de Mérope dont un spectateur admirerait la beauté : «C'était donc de l'imitation que l'on parlait; et pour cela, il fallait se dire à soi-même ; C'est un mensonge ; et tout en le disant, on pleurait et on frémissait. » Article « Illusions », dans Éléments de littérature, ouvr. cité, p. 635.

68. P.-Fr. Godard de Beauchamps, ouvr. cité, p. 29.

69. J.-Fr. Perrin, « Recueillir et transmettre », Féeries, $\mathrm{n}^{\circ}$ 1, 2004, p. 145-171.

70. P.-Fr. Godard de Beauchamps, ouvr. cité, p. 20.

71. Ibid., p. 115-116.

72. Ibid., p. 45.

73. Ibid., p. 28 .

\section{RÉSUMÉS}

Cette étude comparée des contes de Beauchamps (Funestine, 1737), de Sainte-Foy d'Arcq (Le Palais $\mathrm{du}$ silence, conte philosophique, 1754), et de Marmontel (Le Mari Sylphe, 1765) vise à montrer les retentissements physiques et moraux de l'expérience merveilleuse, qui s'apparente ici à une initiation. La palingénésie des trois personnages principaux est la conséquence à la fois de l'intensité des affects provoqués par le phénomène merveilleux et de la démarche herméneutique à laquelle les personnages procèdent pour déchiffrer leur intériorité et le monde qui les entoure. La métatextualité de ces trois contes rapproche, par analogie, l'expérience rapportée par la fiction de celle du lecteur, qui lui aussi vit, à travers la lecture de ces contes à visée philosophique, une sorte d'expérience initiatique.

This comparative study of tales by Beauchamps (Funestine, 1737), Sainte-Foy d'Arcq (Le Palais du silence, conte philosophique, 1754), and Marmontel (Le Mari Sylphe, 1765) aims to show the physical and moral repercussions of the supernatural, which can be likened to a sort of initiation. The palingenesis of the three characters results from both the intensity of the affects caused by the marvelous phenomenon and the hermeneutic reading the characters make of their interiority and the world around them. By analogy, the metatextuality of these three tales makes the fictional experience closer to the reader's personal experience, who also lives, through the reading of these philosophical tales, a kind of initiation. 
INDEX

Keywords : philosophical tale, initiation, marvelous, Beauchamps, Marmontel, Sainte-Foy D'Arcq

Mots-clés : conte philosophique, initiation, merveilleux, Beauchamps, Marmontel, Sainte-Foy D’Arcq

\section{AUTEUR}

MAGALI FOURGNAUD

Université de Bordeaux 